\title{
FROM THE VIRASORO ALGEBRA TO KRICHEVER-NOVIKOV TYPE ALGEBRAS AND BEYOND
}

\author{
MARTIN SCHLICHENMAIER
}

\begin{abstract}
Starting from the Virasoro algebra and its relatives the generalization to higher genus compact Riemann surfaces was initiated by Krichever and Novikov. The elements of these algebras are meromorphic objects which are holomorphic outside a finite set of points. A crucial and non-trivial point is to establish an almost-grading replacing the honest grading in the Virasoro case. Such an almost-grading is given by splitting the set of points of possible poles into two non-empty disjoint subsets. Krichever and Novikov considered the twopoint case. Schlichenmaier studied the most general multi-point situation with arbitrary splittings. Here we will review the path of developments from the Virasoro algebra to its higher genus and multi-point analogs. The starting point will be a Poisson algebra structure on the space of meromorphic forms of all weights. As sub-structures the vector field algebras, function algebras, Lie superalgebras and the related current algebras show up. All these algebras will be almost-graded. In detail almost-graded central extensions are classified. In particular, for the vector field algebra it is essentially unique. The defining cocycle are given in geometric terms. Some applications, including the semi-infinite wedge form representations are recalled. Finally, some remarks on the by Krichever and Sheinman recently introduced Lax operator algebras are made.
\end{abstract}

\section{INTRODUCTION}

Lie groups and Lie algebras are related to symmetries of systems. By the use of the symmetry the system can be better understood, maybe it is even possible to solve it in a certain sense. Here we deal with systems which have an infinite number of independent degrees of freedom. They appear for example in Conformal Field Theory (CFT), see e.g. [2], [67]. But also in the theory of partial differential equations and at many other places in- and outside of mathematics they play an important role. The appearing Lie groups and Lie algebras are infinite dimensional. Some of the simplest nontrivial infinite dimensional Lie algebras are the Witt algebra and its central extension the Virasoro algebra. We will recall their definitions in Section 2 In the sense explained (in particular in CFT) they are related to what

Partial support by the Internal Research Project GEOMQ11, University of Luxembourg, is acknowledged. 
is called the genus zero situation. For CFT on arbitrary genus Riemann surfaces the Krichever-Novikov (KN) type algebras, to be discussed here, will show up as algebras of global symmetry operators.

These algebras are defined via meromorphic objects on compact Riemann surfaces $\Sigma$ of arbitrary genus with controlled polar behaviour. More precisely, poles are only allowed at a fixed finite set of points denoted by $A$. The "classical" examples are the algebras defined by objects on the Riemann sphere (genus zero) with possible poles only at $\{0, \infty\}$. This yields e.g. the Witt algebra, the classical current algebras, including their central extensions the Virasoro, and the affine Kac-Moody algebras [21]. For higher genus, but still only for two points where poles are allowed, they were generalised by Krichever and Novikov [26], [27], [28] in 1987. In 1990 the author [37], [38], [39], [40] extended the approach further to the general multi-point case.

This extension was not a straight-forward generalization. The crucial point is to introduce a replacement of the graded algebra structure present in the "classical" case. Krichever and Novikov found that an almost-grading, see Definition 4.1 below, will be enough to do the usual constructions in representation theory, like triangular decompositions, highest weight modules, Verma modules which are demanded by the applications. In [39], [40] it was realized that a splitting of $A$ into two disjoint non-empty subsets $A=I \cup O$ is crucial for introducing an almost-grading and the corresponding almost-grading was given. In the two-point situation there is only one such splitting (up to inversion) hence there is only one almost-grading, which in the classical case is a honest grading. Similar to the classical situation a Krichever-Novikov algebra, should always be considered as an algebra of meromorphic objects with an almost-grading coming from such a fixed splitting.

I like to point out that already in the genus zero case (i.e. the Riemann sphere case) with more than two points where poles are allowed the algebras will only be almost-graded. In fact, quite a number of interesting new phenomena will show up already there, see [41], [15], [16], [8].

In this review no proofs are supplied. For them I have to refer to the original articles and/or to the forthcoming book [53]. For some applications jointly obtained with Oleg Sheinman, see also [66]. For more on the Witt and Virasoro algebra see for example the book [18].

After recalling the definition of the Witt and Virasoro algebra in Section 2 we start with describing the geometric set-up of Krichever-Novikov (KN) type algebras in Section 3 . We introduce a Poisson algebra structure on the space of meromorphic forms (holomorphic outside of the fixed set $A$ of points where poles are allowed) of all weights (integer and half-integer). Special substructures will yield the function algebra, the vector field algebra and more generally the differential operator algebra. Moreover, we discuss also the Lie superalgebras of KN type defined via forms 
of weight $-1 / 2$. An important example role also is played by the current algebra (arbitrary genus - multi-point) associated to a finite-dimensional Lie algebra.

In Section 4 we introduce the almost-grading induced by the splitting of $A$ into "incoming" and "outgoing" points, $A=I \cup O$.

In Section 5 we discuss central extensions for our algebras. Central extensions appear naturally in the context of quantization and regularization of actions. We give for all our algebras geometrically defined central extensions. The defining cocycle for the Virasoro algebra obviously does not make any sense in the higher genus and/or multi-point case. For the geometric description we use projective and affine connections. In contrast to the classical case there are a many inequivalent cocycles and central extensions. If we restrict our attention to the cases where we can extend the almost-grading to the central extensions the author obtained complete classification and uniqueness results. They are described in Section 5.3.

In Section 6 we present further results. In particular, we discuss how from the representation of the vector field algebra (or more general of the differential operator algebra) on the forms of weight $\lambda$ one obtains semi-infinite wedge representations (fermionic Fock space representations) of the centrally extended algebras. These representations have ground states (vacua), creation and annihilation operators. We add some words about $b-c$ systems, Sugawara construction, Wess-Zumino-Novikov-Witten (WZNW) models, Knizhnik-Zamolodchikov (KZ) connections, and deformations of the Virasoro algebra.

Recently, a new class of current type algebras the Lax operator algebras, were introduced by Krichever and Sheinman [25], [29]. I will report on them in Section7]

In the closing Section 8 some historical remarks (also on related works) on Krichever-Novikov type algebras and some references are given. More references can be found in [53].

\section{The Witt And Virasoro Algebra}

2.1. The Witt Algebra. The Witt algebra $\mathscr{W}$, also sometimes called Virasoro algebra without central term 1 , is the complex Lie algebra generated as vector space by the elements $\left\{e_{n} \mid n \in \mathbb{Z}\right\}$ with Lie structure

$$
\left[e_{n}, e_{m}\right]=(m-n) e_{n+m}, \quad n, m \in \mathbb{Z} .
$$

One of its realization is as complexification of the Lie algebra of polynomial vector fields $\operatorname{Vect}_{\text {pol }}\left(S^{1}\right)$ on the circle $S^{1}$, which is a subalgebra of $\operatorname{Vect}\left(S^{1}\right)$, die Lie algebra of all $C^{\infty}$ vector fields on the circle. In this realization

$$
e_{n}:=-\mathrm{i} \operatorname{expin} \varphi \frac{d}{d \varphi}, \quad n \in \mathbb{Z} .
$$

\footnotetext{
${ }^{1}$ In the book [18] arguments are given why it is more appropriate just to use Virasoro algebra, as Witt introduced "his" algebra in a characteristic $p$ context. Nevertheless, I decided to stick here to the most common convention.
} 
The Lie product is the usual Lie bracket of vector fields.

If we extend these generators to the whole punctured complex plane we obtain

$$
e_{n}=z^{n+1} \frac{d}{d z}, \quad n \in \mathbb{Z} .
$$

This gives another realization of the Witt algebra as the algebra of those meromorphic vector fields on the Riemann sphere $\mathbb{P}^{1}(\mathbb{C})$ which are holomorphic outside $\{0\}$ and $\{\infty\}$.

Let $z$ be the (quasi) global coordinate $z$ (quasi, because it is not defined at $\infty$ ). Let $w=1 / z$ be the local coordinate at $\infty$. A global meromorphic vector field $v$ on $\mathbb{P}^{1}(\mathbb{C})$ will be given on the corresponding subsets where $z$ resp. $w$ are defined as

$$
v=\left(v_{1}(z) \frac{d}{d z}, v_{2}(w) \frac{d}{d w}\right), \quad v_{2}(w)=-v_{1}(z(w)) w^{2} .
$$

The function $v_{1}$ will determine the vector field $v$. Hence, we will usually just write $v_{1}$ and in fact identify the vector field $v$ with its local representing function $v_{1}$, which we will denote by the same letter.

For the bracket we calculate

$$
[v, u]=\left(v \frac{d}{d z} u-u \frac{d}{d z} v\right) \frac{d}{d z}
$$

The space of all meromorphic vector fields constitute a Lie algebra. The subspace of those meromorphic vector fields which are holomorphic outside of $\{0, \infty\}$ is a Lie subalgebra. Its elements can be given as

$$
v(z)=f(z) \frac{d}{d z}
$$

where $f$ is a meromorphic function on $\mathbb{P}^{1}(\mathbb{C})$, which is holomorphic outside $\{0, \infty\}$. Those are exactly the Laurent polynomials $\mathbb{C}\left[z, z^{-1}\right]$. Consequently, this subalgebra has the set $\left\{e_{n}, n \in \mathbb{Z}\right\}$ as basis elements. The Lie product is the same and it can be identified with the Witt algebra $\mathscr{W}$.

The subalgebra of global holomorphic vector fields is $\left\langle e_{-1}, e_{0}, e_{1}\right\rangle_{\mathbb{C}}$. It is isomorphic to the Lie algebra $\mathfrak{s l}(2, \mathbb{C})$.

The algebra $\mathscr{W}$ is more than just a Lie algebra. It is a graded Lie algebra. If we set for the degree $\operatorname{deg}\left(e_{n}\right):=n$ then $\operatorname{deg}\left(\left[e_{n}, e_{m}\right]\right)=\operatorname{deg}\left(e_{n}\right)+\operatorname{deg}\left(e_{m}\right)$ and we obtain the degree decomposition

$$
\mathscr{W}=\bigoplus_{n \in \mathbb{Z}} \mathscr{W}_{n}, \quad \mathscr{W}_{n}=\left\langle e_{n}\right\rangle_{\mathbb{C}} .
$$

Note that $\left[e_{0}, e_{n}\right]=n e_{n}$, which says that the degree decomposition is the eigen-space decomposition with respect to the adjoint action of $e_{0}$ on $\mathscr{W}$.

Algebraically $\mathscr{W}$ can also be given as Lie algebra of derivations of the algebra of Laurent polynomials $\mathbb{C}\left[z, z^{-1}\right]$. 
2.2. The Virasoro Algebra. In the process of quantizing or regularization one is often forced to modify an action of a Lie algebra. A typical example is given by the product of infinite sums of operators. Quite often they are only well-defined if a certain "normal ordering" is introduced. In this way the modified action will only be a projective action. This can be made to an honest Lie action by passing to a suitable central extension of the Lie algebra.

For the Witt algebra the universal one-dimensional central extension is the Virasoro algebra $\mathscr{V}$. As vector space it is the direct sum $\mathscr{V}=\mathbb{C} \oplus \mathscr{W}$. If we set for $x \in \mathscr{W}, \hat{x}:=(0, x)$, and $t:=(1,0)$ then its basis elements are $\hat{e}_{n}, n \in \mathbb{Z}$ and $t$ with the Lie product

$$
\left[\hat{e}_{n}, \hat{e}_{m}\right]=(m-n) \hat{e}_{n+m}-\frac{1}{12}\left(n^{3}-n\right) \delta_{n}^{-m} t, \quad\left[\hat{e}_{n}, t\right]=[t, t]=0,
$$

forl all $n, m \in \mathbb{Z}$. If we set $\operatorname{deg}\left(\hat{e}_{n}\right):=\operatorname{deg}\left(e_{n}\right)=n$ and $\operatorname{deg}(t):=0$ then $\mathscr{V}$ becomes a graded algebra. The algebra $\mathscr{W}$ will only be a subspace, not a subalgebra of $\mathscr{V}$. It will be a quotient. In some abuse of notation we identify the element $\hat{x} \in \mathscr{V}$ with $x \in \mathscr{W}$. Up to equivalence and rescaling the central element $t$, this is beside the trivial (splitting) central extension the only central extension.

\section{The KricheVer-Novikov Type Algebras}

3.1. The Geometric Set-Up. For the whole article let $\Sigma$ be a compact Riemann surface without any restriction for the genus $g=g(\Sigma)$. Furthermore, let $A$ be a finite subset of $\Sigma$. Later we will need a splitting of $A$ into two non-empty disjoint subsets $I$ and $O$, i.e. $A=I \cup O$. Set $N:=\# A, K:=\# I, M:=\# O$, with $N=K+M$. More precisely, let

$$
I=\left(P_{1}, \ldots, P_{K}\right), \quad \text { and } \quad O=\left(Q_{1}, \ldots, Q_{M}\right)
$$

be disjoint ordered tuples of distinct points ("marked points", "punctures") on the Riemann surface. In particular, we assume $P_{i} \neq Q_{j}$ for every pair $(i, j)$. The points in $I$ are called the in-points, the points in $O$ the out-points. Sometimes we consider $I$ and $O$ simply as sets.

In the article we sometimes refer to the classical situation. By this we understand

$$
\Sigma=\mathbb{P}^{1}(\mathbb{C})=S^{2}, \quad I=\{z=0\}, \quad O=\{z=\infty\}
$$

The following Figures 1,2, 3 exemplify the different situations:

Our objects, algebras, structures, ... will be meromorphic objects defined on $\Sigma$ which are holomorphic outside of the points in $A$. To introduce the objects let $\mathscr{K}=\mathscr{K}_{\Sigma}$ be the canonical line bundle of $\Sigma$, resp. the locally free canonically sheaf. The local sections of the bundle are the local holomorphic differentials. If $P \in \Sigma$ is a point and $z$ a local holomorphic coordinate at $P$ then a local holomorphic differential can be written as $f(z) d z$ with a local holomorphic function $f$ defined in a

\footnotetext{
${ }^{2}$ Here $\delta_{k}^{l}$ is the Kronecker delta which is equal to 1 if $k=l$, otherwise zero.
} 


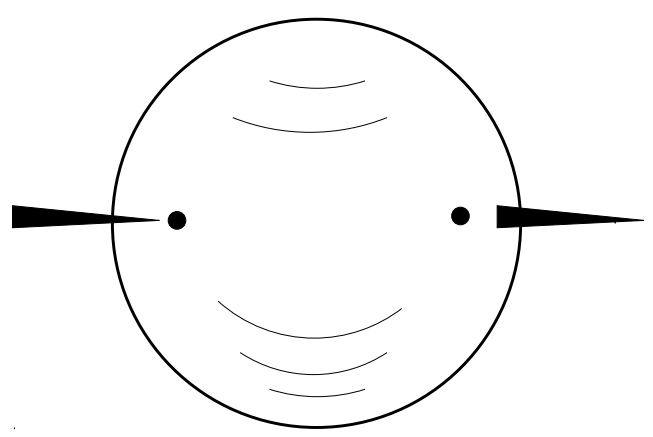

FIGURE 1. Riemann surface of genus zero with one incoming and one outgoing point.

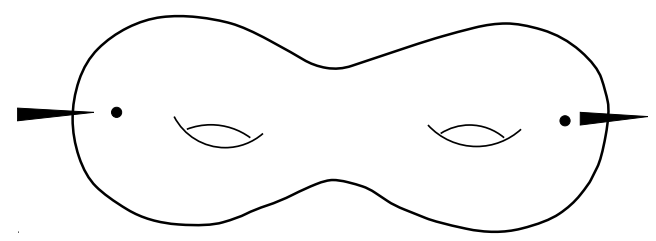

FIGURE 2. Riemann surface of genus two with one incoming and one outgoing point.

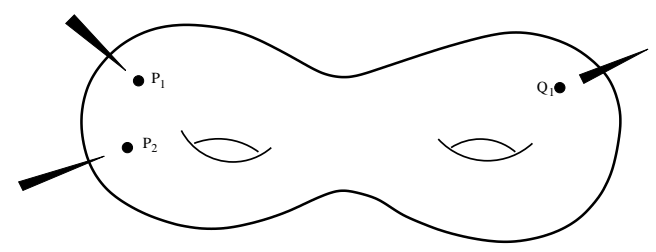

FIGURE 3. Riemann surface of genus two with two incoming points and one outgoing point.

neighbourhood of $P$. A global holomorphic section can be described locally with respect to a covering by coordinate charts $\left(U_{i}, z_{i}\right)_{i \in J}$ by a system of local holomorphic functions $\left(f_{i}\right)_{i \in J}$, which are related by the transformation rule induced by the coordinate change map $z_{j}=z_{j}\left(z_{i}\right)$ and the condition $f_{i} d z_{i}=f_{j} d z_{j}$ yielding

$$
f_{j}=f_{i} \cdot\left(\frac{d z_{j}}{d z_{i}}\right)^{-1} .
$$

Moreover, a meromorphic section of $\mathscr{K}$ is given as a collection of local meromorphic functions $\left(h_{i}\right)_{i \in J}$ for which the transformation law (11) still is true. 
In the following $\lambda$ is either an integer or a half-integer. If $\lambda$ is an integer then (1) $\mathscr{K}^{\lambda}=\mathscr{K}^{\otimes \lambda}$ for $\lambda>0$,

(2) $\mathscr{K}^{0}=\mathscr{O}$, the trivial line bundle, and

(3) $\mathscr{K}^{\lambda}=\left(\mathscr{K}^{*}\right)^{\otimes(-\lambda)}$ for $\lambda<0$.

Here as usual $\mathscr{K}^{*}$ denotes the dual line bundle to the canonical line bundle. The dual line bundle is the holomorphic tangent line bundle, whose local sections are the holomorphic tangent vector fields $f(z)(d / d z)$. If $\lambda$ is a half-integer, then we first have to fix a "square root" of the canonical line bundle, sometimes called a theta-characteristics. This means we fix a line bundle $L$ for which $L^{\otimes 2}=\mathscr{K}$.

After such a choice of $L$ is done we set $\mathscr{K}^{\lambda}=\mathscr{K}_{L}^{\lambda}=L^{\otimes 2 \lambda}$. In most cases we will drop the mentioning of $L$, but we have to keep the choice in mind. Also the fine-structure of the algebras we are about to define will depend on the choice. But the main properties will remain the same.

Remark 3.1. A Riemann surface of genus $g$ has exactly $2^{2 g}$ non-isomorphic square roots of $\mathscr{K}$. For $g=0$ we have $\mathscr{K}=\mathscr{O}(-2)$, and $L=\mathscr{O}(-1)$, the tautological bundle, is the unique square root. Already for $g=1$ we have 4 non-isomorphic ones. As in this case $\mathscr{K}=\mathscr{O}$ one solution is $L_{0}=\mathscr{O}$. But we have also other bundles $L_{i}, i=1,2,3$. Note that $L_{0}$ has a non-vanishing global holomorphic section, whereas this is not the case for $L_{1}, L_{2}, L_{3}$. In general, depending on the parity of $\operatorname{dim} \mathrm{H}(\Sigma, L)$, one distinguishes even and odd theta characteristics $L$. For $g=1$ the bundle $\mathscr{O}$ is an odd, the others are even theta characteristics.

We set

$$
\begin{array}{r}
\mathscr{F}^{\lambda}:=\mathscr{F}^{\lambda}(A):=\left\{f \text { is a global meromorphic section of } K^{\lambda} \mid\right. \\
\text { such that } f \text { is holomorphic over } \Sigma \backslash A\} .
\end{array}
$$

We will drop the set $A$ in the notation. Obviously, $\mathscr{F}^{\lambda}$ is an infinite dimensional $\mathbb{C}$-vector space. Recall that in the case of half-integer $\lambda$ everything depends on the theta characteristic $L$.

The elements of the space $\mathscr{F}^{\lambda}$ we call meromorphic forms of weight $\lambda$ (with respect to the theta characteristic $L$ ). In local coordinates $z_{i}$ we can write such a form as $f_{i} d z_{i}^{\lambda}$, with $f_{i}$ a local holomorphic, resp. meromorphic form.

Special important cases of the weights are the functions $(\lambda=0)$, the space is also denoted by $\mathscr{A}$, the vector fields $(\lambda=-1)$, denoted by $\mathscr{L}$, the differentials $(\lambda=1)$, and the quadratic differentials $(\lambda=2)$.

Next we introduce algebraic operations on the space of all weights

$$
\mathscr{F}:=\bigoplus_{\lambda \in \frac{1}{2} \mathbb{Z}} \mathscr{F}^{\lambda} .
$$

These operations will allow us to introduce the algebras we are heading for. 
3.2. Associative Structure. The natural map of the locally free sheaves of rang one

$$
\mathscr{K}^{\lambda} \times \mathscr{K}^{v} \rightarrow \mathscr{K}^{\lambda} \otimes \mathscr{K}^{v} \cong \mathscr{K}^{\lambda+v}, \quad(s, t) \mapsto s \otimes t,
$$

defines a bilinear map

$$
\cdot: \mathscr{F}^{\lambda} \times \mathscr{F}^{v} \rightarrow \mathscr{F}^{\lambda+v}
$$

With respect to local trivialisations this corresponds to the multiplication of the local representing meromorphic functions

$$
\left(s d z^{\lambda}, t d z^{v}\right) \mapsto s d z^{\lambda} \cdot t d z^{v}=s \cdot t d z^{\lambda+v} .
$$

If there is no danger of confusion then we will mostly use the same symbol for the section and for the local representing function.

The following is obvious

Proposition 3.2. The vector space $\mathscr{F}$ is an associative and commutative graded (over $\frac{1}{2} \mathbb{Z}$ ) algebras. Moreover, $\mathscr{A}=\mathscr{F}^{0}$ is a subalgebra.

Definition 3.3. The associative algebra $\mathscr{A}$ is the Krichever-Novikov function algebra (associated to $(\Sigma, A)$ ).

Of course, it is the algebra of meromorphic functions on $\Sigma$ which are holomorphic outside of $A$. The spaces $\mathscr{F}^{\lambda}$ are modules over $\mathscr{A}$. In the classical situation $\mathscr{A}=$ $\mathbb{C}\left[z, z^{-1}\right]$, the algebra of Laurent polynomials.

3.3. Lie Algebra Structure. Next we define a Lie algebra structure on the space $\mathscr{F}$. The structure is induced by the map

$$
\mathscr{F}^{\lambda} \times \mathscr{F}^{v} \rightarrow \mathscr{F}^{\lambda+v+1}, \quad(s, t) \mapsto[s, t],
$$

which is defined in local representatives of the sections by

$$
\left(s d z^{\lambda}, t d z^{v}\right) \mapsto\left[s d z^{\lambda}, t d z^{\nu}\right]:=\left((-\lambda) s \frac{d t}{d z}+v t \frac{d s}{d z}\right) d z^{\lambda+v+1},
$$

and bilinearly extended to $\mathscr{F}$.

Proposition 3.4. [43], [53]

(a) The bilinear map $[.,$.$] defines a Lie algebra structure on \mathscr{F}$.

(b) The space $\mathscr{F}$ with respect to $\cdot$ and $[.,$.$] is a Poisson algebra.$

Next we consider certain important substructures.

3.4. The Vector Field Algebra and the Lie Derivative. For $\lambda=v=-1$ in 17 we end up in $\mathscr{F}^{-1}$ again. Hence,

Proposition 3.5. The subspace $\mathscr{L}=\mathscr{F}^{-1}$ is a Lie subalgebra, and the $\mathscr{F}^{\lambda}$ 's are Lie modules over $\mathscr{L}$. 
As forms of weight -1 are vector fields, $\mathscr{L}$ could also be defined as the Lie algebra of those meromorphic vector fields on the Riemann surface $\Sigma$ which are holomorphic outside of $A$. The product (18) gives the usual Lie bracket of vector fields and the Lie derivative for their actions on forms. Due to its importance let us specialize this. We obtain (naming the local functions with the same symbol as the section)

$$
\begin{gathered}
{[e, f]_{\mid}(z)=\left[e(z) \frac{d}{d z}, f(z) \frac{d}{d z}\right]=\left(e(z) \frac{d f}{d z}(z)-f(z) \frac{d e}{d z}(z)\right) \frac{d}{d z}} \\
\nabla_{e}(g)_{\mid}(z)=L_{e}(g)_{\mid}=e \cdot g_{\mid}=\left(e(z) \frac{d g}{d z}(z)+\lambda g(z) \frac{d e}{d z}(z)\right)(d z)^{\lambda} .
\end{gathered}
$$

Definition 3.6. The algebra $\mathscr{L}$ is called Krichever-Novikov type vector field algebra (associated to $(\Sigma, A)$.

In the classical case this gives the Witt algebra.

3.5. The Algebra of Differential Operators. In $\mathscr{F}$, considered as Lie algebra, $\mathscr{A}=\mathscr{F}^{0}$ is an abelian Lie subalgebra and the vector space sum $\mathscr{F}^{0} \oplus \mathscr{F}^{-1}=\mathscr{A} \oplus$ $\mathscr{L}$ is also a Lie subalgebra of $\mathscr{F}$. In an equivalent way it can also be constructed as semi-direct sum of $\mathscr{A}$ considered as abelian Lie algebra and $\mathscr{L}$ operating on $\mathscr{A}$ by taking the derivative.

Definition 3.7. This Lie algebra is called the Lie algebra of differential operators of degree $\leq 1$ of $\mathrm{KN}$ type (associated to $(\Sigma, A)$ ) and is denoted by $\mathscr{D}^{1}$.

In more direct terms $\mathscr{D}^{1}=\mathscr{A} \oplus \mathscr{L}$ as vector space direct sum and endowed with the Lie product

$$
[(g, e),(h, f)]=(e \cdot h-f \cdot g,[e, f]) .
$$

The spaces $\mathscr{F}^{\lambda}$ will be Lie-modules over $\mathscr{D}^{1}$.

Its universal enveloping algebra will be the algebra of all differential operators of arbitrary degree [40], [42], [46].

3.6. The Superalgebra of Half Forms. Next we consider the associative product

$$
\cdot \mathscr{F}^{-1 / 2} \times \mathscr{F}^{-1 / 2} \rightarrow \mathscr{F}^{-1}=\mathscr{L} .
$$

We introduce the vector space and the product

$$
\mathscr{S}:=\mathscr{L} \oplus \mathscr{F}^{-1 / 2}, \quad[(e, \varphi),(f, \psi)]:=([e, f]+\varphi \cdot \psi, e \cdot \varphi-f \cdot \psi) .
$$

Usually we will denote the elements of $\mathscr{L}$ by $e, f, \ldots$, and the elements of $\mathscr{F}^{-1 / 2}$ by $\varphi, \psi, \ldots$.

The definition (23) can be reformulated as an extension of [.,.] on $\mathscr{L}$ to a "superbracket" (denoted by the same symbol) on $\mathscr{S}$ by setting

$$
[e, \varphi]:=-[\varphi, e]:=e \cdot \varphi=\left(e \frac{d \varphi}{d z}-\frac{1}{2} \varphi \frac{d e}{d z}\right)(d z)^{-1 / 2}
$$


and

$$
[\varphi, \psi]:=\varphi \cdot \psi
$$

We call the elements of $\mathscr{L}$ elements of even parity, and the elements of $\mathscr{F}^{-1 / 2}$ elements of odd parity. For such elements $x$ we denote by $\bar{x} \in\{\overline{0}, \overline{1}\}$ their parity.

The sum (23) can also be described as $\mathscr{S}=\mathscr{S}_{\overline{0}} \oplus \mathscr{S}_{\overline{1}}$, where $\mathscr{S}_{\bar{i}}$ is the subspace of elements of parity $\bar{i}$.

Proposition 3.8. [52] The space $\mathscr{S}$ with the above introduced parity and product is a Lie superalgebra.

Definition 3.9. The algebra $\mathscr{S}$ is the Krichever-Novikov type Lie superalgebra (associated to $(\Sigma, A)$ ).

Classically this Lie superalgebra corresponds to the Neveu-Schwarz superalgebra. See in this context also [10], [3], [5].

3.7. Jordan Superalgebra. Leidwanger and Morier-Genoux introduced in [30] a Jordan superalgebra in the Krichever-Novikov setting, i.e.

$$
\mathscr{J}:=\mathscr{F}^{0} \oplus \mathscr{F}^{-1 / 2}=\mathscr{J}_{\overline{0}} \oplus \mathscr{J}_{\overline{1}} .
$$

Recall that $\mathscr{A}=\mathscr{F}^{0}$ is the associative algebra of meromorphic functions. They define the (Jordan) product $\circ$ is via the algebra structures for the spaces $\mathscr{F}^{\lambda}$ by

$$
\begin{aligned}
& f \circ g:=f \cdot g \in \mathscr{F}^{0}, \\
& f \circ \varphi:=f \cdot \varphi \quad \in \mathscr{F}^{-1 / 2} \\
& \varphi \circ \psi:=[\varphi, \psi] \quad \in \mathscr{F}^{0} .
\end{aligned}
$$

By rescaling the second definition with the factor $1 / 2$ one obtains a Lie antialgebra. See [30] for more details and additional results on representations.

3.8. Current Algebras. We start with $\mathfrak{g}$ a complex finite-dimensional Lie algebra and endow the tensor product $\overline{\mathfrak{g}}=\mathfrak{g} \otimes_{\mathbb{C}} \mathscr{A}$ with the Lie bracket

$$
[x \otimes f, y \otimes g]=[x, y] \otimes f \cdot g, \quad x, y \in \mathfrak{g}, \quad f, g \in \mathscr{A} .
$$

The algebra $\overline{\mathfrak{g}}$ is the higher genus current algebra. It is an infinite dimensional Lie algebra and might be considered as the Lie algebra of $\mathfrak{g}$-valued meromorphic functions on the Riemann surface with poles only outside of $A$. Note that we allow also the case of $\mathfrak{g}$ an abelian Lie algebra.

Definition 3.10. The algebra $\overline{\mathfrak{g}}$ is called current algebra of Krichever Novikov type (associated to $(\Sigma, A)$ ).

Sometimes also the name loop algebra is used.

In the classical case the current algebra $\overline{\mathfrak{g}}$ is the standard current algebra $\overline{\mathfrak{g}}=$ $\mathfrak{g} \otimes \mathbb{C}\left[z^{-1}, z\right]$ with Lie bracket

$$
\left[x \otimes z^{n}, y \otimes z^{m}\right]=[x, y] \otimes z^{n+m} \quad x, y \in \mathfrak{g}, \quad n, m \in \mathbb{Z} .
$$


To point out the dependence on the geometrical structure we added always "(associated to $(\Sigma, A))$ in the definition. For simplicity we will drop it starting from now.

\section{Almost-Graded Structure}

4.1. Definition of Almost-Gradedness. Recall the classical situation. This is the Riemann surface $\mathbb{P}^{1}(\mathbb{C})=S^{2}$, i.e. the Riemann surface of genus zero, and the points where poles are allowed are $\{0, \infty\}$ ). In this case the algebras introduced in the last section are graded algebras. In the higher genus case and even in the genus zero case with more than two points where poles are allowed there is no non-trivial grading anymore. As realized by Krichever and Novikov [26] there is a weaker concept, an almost-grading, which to a large extend is a valuable replacement of a honest grading. Such an almost-grading is induced by a splitting of the set $A$ into two nonempty and disjoint sets $I$ and $O$. The almost-grading is fixed by exhibiting certain basis elements in the spaces $\mathscr{F}^{\lambda}$ as homogeneous.

Definition 4.1. Let $\mathscr{L}$ be a Lie or an associative algebra such that $\mathscr{L}=\oplus_{n \in \mathbb{Z}} \mathscr{L}_{n}$ is a vector space direct sum, then $\mathscr{L}$ is called an almost-graded (Lie-) algebra if

(i) $\operatorname{dim} \mathscr{L}_{n}<\infty$,

(ii) There exists constants $L_{1}, L_{2} \in \mathbb{Z}$ such that

$$
\mathscr{L}_{n} \cdot \mathscr{L}_{m} \subseteq \bigoplus_{h=n+m-L_{1}}^{n+m+L_{2}} \mathscr{L}_{h}, \quad \forall n, m \in \mathbb{Z} .
$$

The elements in $\mathscr{L}_{n}$ are called homogeneous elements of degree $n$, and $\mathscr{L}_{n}$ is called homogeneous subspace of degree $n$.

If $\operatorname{dim} \mathscr{L}_{n}$ is bounded with a bound independent of $n$ we call $\mathscr{L}$ strongly almostgraded. If we drop the condition that $\operatorname{dim} \mathscr{L}_{n}$ is finite we call $\mathscr{L}$ weakly almostgraded.

In a similar manner almost-graded modules over almost-graded algebras are defined. We can extend in an obvious way the definition to superalgebras, resp. even to more general algebraic structures. This definition makes complete sense also for more general index sets $\mathbb{J}$. In fact we will consider the index set $\mathbb{J}=(1 / 2) \mathbb{Z}$ in the case of superalgebras. The even elements (with respect to the super-grading) will have integer degree, the odd elements half-integer degree.

4.2. Separating Cycle and Krichever-Novikov Duality. Let $C_{i}$ be positively oriented (deformed) circles around the points $P_{i}$ in $I, i=1, \ldots, K$ and $C_{j}^{*}$ positively oriented ones around the points $Q_{j}$ in $O, j=1, \ldots, M$.

A cycle $C_{S}$ is called a separating cycle if it is smooth, positively oriented of multiplicity one and if it separates the in- from the out-points. It might have multiple components. In the following we will integrate meromorphic differentials on $\Sigma$ 
without poles in $\Sigma \backslash A$ over closed curves $C$. Hence, we might consider the $C$ and $C^{\prime}$ as equivalent if $[C]=\left[C^{\prime}\right]$ in $\mathrm{H}(\Sigma \backslash A, \mathbb{Z})$. In this sense we can write for every separating cycle

$$
\left[C_{S}\right]=\sum_{i=1}^{K}\left[C_{i}\right]=-\sum_{j=1}^{M}\left[C_{j}^{*}\right]
$$

The minus sign appears due to the opposite orientation. Another way for giving such a $C_{S}$ is via level lines of a "proper time evolution", for which I refer to Ref. [39].

Given such a separating cycle $C_{S}$ (resp. cycle class) we define a linear map

$$
\mathscr{F}^{1} \rightarrow \mathbb{C}, \quad \omega \mapsto \frac{1}{2 \pi \mathrm{i}} \int_{C_{S}} \omega .
$$

As explained above the map will not depend on the separating line $C_{S}$ chosen, as two of such will be homologous and the poles of $\omega$ are only located in $I$ and $O$.

Consequently, the integration of $\omega$ over $C_{S}$ can also be described over the special cycles $C_{i}$ or equivalently over $C_{j}^{*}$. This integration corresponds to calculating residues

$$
\omega \mapsto \frac{1}{2 \pi \mathrm{i}} \int_{C_{S}} \omega=\sum_{i=1}^{K} \operatorname{res}_{P_{i}}(\omega)=-\sum_{l=1}^{M} \operatorname{res}_{Q_{l}}(\omega) .
$$

Definition 4.2. The pairing

$$
\mathscr{F}^{\lambda} \times \mathscr{F}^{1-\lambda} \rightarrow \mathbb{C}, \quad(f, g) \mapsto\langle f, g\rangle:=\frac{1}{2 \pi \mathrm{i}} \int_{C_{S}} f \cdot g,
$$

between $\lambda$ and $1-\lambda$ forms is called Krichever-Novikov (KN) pairing.

Note that the pairing depends not only on $A$ (as the $\mathscr{F}^{\lambda}$ depend on it) but also critically on the splitting of $A$ into $I$ and $O$ as the integration path will depend on it. Once the splitting is fixed the pairing will be fixed too.

By exhibiting dual basis elements further down we will see that it is non-degenerate.

4.3. The Homogeneous Subspaces. Depending on whether $\lambda$ is integer or halfinteger we set $\mathbb{J}_{\lambda}=\mathbb{Z}$ or $\mathbb{J}_{\lambda}=\mathbb{Z}+1 / 2$. For $\mathscr{F} \lambda$ we introduce for $m \in \mathbb{J}_{\lambda}$ subspaces $\mathscr{F}_{m}^{\lambda}$ of dimension $K$, where $K=\# I$, by exhibiting certain elements $f_{m, p}^{\lambda} \in \mathscr{F}^{\lambda}, p=$ $1, \ldots, K$ which constitute a basis of $\mathscr{F}_{m}^{\lambda}$. Recall that the spaces $\mathscr{F}^{\lambda}$ for $\lambda \in \mathbb{Z}+1 / 2$ depend on the chosen square root $L$ (the theta characteristic) of $\mathscr{K}$. The elements are the elements of degree $m$. As explained in the following, the degree is in an essential way related to the zero orders of the elements at the points in $I$.

Let $I=\left\{P_{1}, P_{2}, \ldots, P_{K}\right\}$ then we have for the zero-order at the point $P_{i} \in I$ of the element $f_{n, p}^{\lambda}$

$$
\operatorname{ord}_{P_{i}}\left(f_{n, p}^{\lambda}\right)=(n+1-\lambda)-\delta_{i}^{p}, \quad i=1, \ldots, K .
$$

The prescription at the points in $O$ is made in such a way that the element $f_{m, p}^{\lambda}$ is essentially uniquely given. Essentially unique means up to multiplication with 
a constan 3 . After fixing as additional geometric data a system of coordinates $z_{l}$ centered at $P_{l}$ for $l=1, \ldots, K$ and requiring that

$$
f_{n, p}^{\lambda}\left(z_{p}\right)=z_{p}^{n-\lambda}\left(1+O\left(z_{p}\right)\right)\left(d z_{p}\right)^{\lambda}
$$

the element $f_{n, p}$ is uniquely fixed. In fact, the element $f_{n, p}^{\lambda}$ only depends on the first jet of the coordinate $z_{p}[55]$.

Example. Here we will not give the general recipe for the prescription at the points in $O$, see [39], [40], [53]. Just to give an example which is also an important special case, assume $O=\{Q\}$ is a one-element set. If either the genus $g=0$, or $g \geq 2$, $\lambda \neq 0,1 / 2,1$ and the points in $A$ are in generic position then we require

$$
\operatorname{ord}_{Q}\left(f_{n, p}^{\lambda}\right)=-K \cdot(n+1-\lambda)+(2 \lambda-1)(g-1) .
$$

In the other cases (e.g. for $g=1$ ) there are some modifications at the point in $O$ necessary for finitely many $n$.

Theorem 4.3. [39], [40], [53] Set

$$
\mathscr{B}^{\lambda}:=\left\{f_{n, p}^{\lambda} \mid n \in \mathbb{J}_{\lambda}, p=1, \ldots, K\right\} .
$$

Then (a) $\mathscr{B}^{\lambda}$ is a basis of the vector space $\mathscr{F}^{\lambda}$.

(b) The introduced basis $\mathscr{B}^{\lambda}$ of $\mathscr{F}^{\lambda}$ and $\mathscr{B}^{1-\lambda}$ of $\mathscr{F}^{1-\lambda}$ are dual to each other with respect to the Krichever-Novikov pairing (34), i.e.

$$
\left\langle f_{n, p}^{\lambda}, f_{-m, r}^{1-\lambda}\right\rangle=\delta_{p}^{r} \delta_{n}^{m}, \quad \forall n, m \in \mathbb{J}_{\lambda}, \quad r, p=1, \ldots, K .
$$

From part (b) of the theorem it follows that the Krichever-Novikov pairing is non-degenerate. Moreover, any element $v \in \mathscr{F}^{1-\lambda}$ acts as linear form on $\mathscr{F}^{\lambda}$ via

$$
\Phi_{v}: \mathscr{F}^{\lambda} \mapsto \mathbb{C}, \quad w \mapsto \Phi_{v}(w):=\langle v, w\rangle .
$$

Via this pairing $\mathscr{F}^{1-\lambda}$ can be considered as subspace of $\left(\mathscr{F}^{\lambda}\right)^{*}$. But I like to stress the fact that the identification depends on the splitting of $A$ into $I$ and $O$ as the KN pairing depends on it.

The full space $\left(\mathscr{F}^{\lambda}\right)^{*}$ can even be described with the help of the pairing. Consider the series

$$
\hat{v}:=\sum_{m \in \mathbb{Z}} \sum_{p=1}^{K} a_{m, p} f_{m, p}^{1-\lambda}
$$

as a formal series, then $\Phi_{\hat{v}}$ (as a distribution) is a well-defined element of $\mathscr{F}^{\lambda^{*}}$, as it will be only evaluated for finitely many basis elements in $\mathscr{F}^{\lambda}$. Vice versa, every

\footnotetext{
${ }^{3}$ Strictly speaking, there are some special cases where some constants have to be added such that the Krichever-Novikov duality [39] is valid, see [39].
} 
element of $\mathscr{F}^{\lambda^{*}}$ can be given by a suitable $\hat{v}$. Every $\phi \in\left(\mathscr{F}^{\lambda}\right)^{*}$ is uniquely given by the scalars $\phi\left(f_{m, r}^{\lambda}\right)$. We set

$$
\hat{v}:=\sum_{m \in \mathbb{Z}} \sum_{p=1}^{K} \phi\left(f_{-m, p}^{\lambda}\right) f_{m, p}^{1-\lambda} .
$$

Obviously, $\Phi_{\hat{v}}=\phi$. For more information about this "distribution interpretation" see [40], [43].

The dual elements of $\mathscr{L}$ will be given by the formal series (41) with basis elements from $\mathscr{F}^{2}$ the quadratic differentials, the dual elements of $\mathscr{A}$ correspondingly from $\mathscr{F}^{1}$ the differentials, and the dual elements of $\mathscr{F}^{-1 / 2}$ correspondingly from $\mathscr{F}^{3 / 2}$. The spaces $\mathscr{F}^{2}, \mathscr{F}^{1}$ and $\mathscr{F}^{3 / 2}$ themselves can be considered as some kind of restricted duals.

It is quite convenient to use special notations for elements of some important weights:

$$
\begin{gathered}
e_{n, p}:=f_{n, p}^{-1}, \quad \varphi_{n, p}:=f_{n, p}^{-1 / 2}, \quad A_{n, p}:=f_{n, p}^{0}, \\
\omega^{n, p}:=f_{-n, p}^{1}, \quad \Omega^{n, p}:=f_{-n, p}^{2} .
\end{gathered}
$$

In view of (39) for the forms of weight 1 and 2 it is convenient to invert the index $n$ and write it as a superscript.

\subsection{The Algebras.}

Theorem 4.4. [39], [40], [53] There exists constants $R_{1}$ and $R_{2}$ (depending on the genus $g$, and on the number and splitting of the points in $A$ ) independent of $n, m \in \mathbb{J}$ such that for the basis elements

$$
\begin{aligned}
f_{n, p}^{\lambda} \cdot f_{m, r}^{v}=f_{n+m, r}^{\lambda+v} \delta_{p}^{r} & \\
& +\sum_{h=n+m+1}^{n+m+R_{1}} \sum_{s=1}^{K} a_{(n, p)(m, r)}^{(h, s)} f_{h, s}^{\lambda+v}, \quad a_{(n, p)(m, r)}^{(h, s)} \in \mathbb{C}, \\
{\left[f_{n, p}^{\lambda}, f_{m, r}^{v}\right]=} & (-\lambda m+v n) f_{n+m, r}^{\lambda+v+1} \delta_{p}^{r} \\
& +\sum_{h=n+m+1}^{n+m+R_{2}} \sum_{s=1}^{K} b_{(n, p)(m, r)}^{(h, s)} f_{h, s}^{\lambda+v+1}, \quad b_{(n, p)(m, r)}^{(h, s)} \in \mathbb{C} .
\end{aligned}
$$

This says in particular that with respect to both the associative and Lie structure the algebra $\mathscr{F}$ is weakly almost-graded. In generic situations and for $N=2$ points one obtains $R_{1}=g$ and $R_{2}=3 g$.

The reason why we only have weakly almost-gradedness is that

$$
\mathscr{F}^{\lambda}=\bigoplus_{m \in \mathbb{J}_{\lambda}} \mathscr{F}_{m}^{\lambda}, \quad \text { with } \quad \operatorname{dim} \mathscr{F}_{m}^{\lambda}=K
$$


If we add up for a fixed $m$ all $\lambda$ we get that our homogeneous spaces are infinite dimensional.

In the definition of our KN type algebra only finitely many $\lambda$ are involved, hence the following is immediate

Theorem 4.5. The Krichever-Novikov type vector field algebras $\mathscr{L}$, function algebras $\mathscr{A}$, differential operator algebras $\mathscr{D}^{1}$, Lie superalgebras $\mathscr{S}$, and Jordan superalgebras $\mathscr{J}$ are all (strongly) almost-graded.

We obtain

$$
\operatorname{dim} \mathscr{L}_{n}=\operatorname{dim} \mathscr{A}_{n}=K, \quad \operatorname{dim} \mathscr{S}_{n}=\operatorname{dim} \mathscr{J}_{n}=2 K, \quad \operatorname{dim} \mathscr{D}_{n}^{1}=3 K .
$$

If $\mathscr{U}$ is one of these algebras, with product denoted by [, ] then

$$
\left[\mathscr{U}_{n}, \mathscr{U}_{m}\right] \subseteq \bigoplus_{h=n+m}^{n+m+R_{i}} \mathscr{U}_{h}
$$

with $R_{i}=R_{1}$ for $\mathscr{U}=\mathscr{A}$ and $R_{i}=R_{2}$ otherwise.

For further reference let us specialize the lowest degree term component in (44) for certain special cases.

$$
\begin{aligned}
A_{n, p} \cdot A_{m, r} & =A_{n+m, r} \delta_{r}^{p}+\text { h.d.t. } \\
A_{n, p} \cdot f_{m, r}^{\lambda} & =f_{n+m, r}^{\lambda} \delta_{r}^{p}+\text { h.d.t. } \\
{\left[e_{n, p}, e_{m, r}\right] } & =(m-n) \cdot e_{n+m, r} \delta_{r}^{p}+\text { h.d.t. } \\
e_{n, p} \cdot f_{m, r}^{\lambda} & =(m+n \lambda) \cdot f_{n+m, r}^{\lambda} \delta_{r}^{p}+\text { h.d.t. }
\end{aligned}
$$

Here h.d.t. denote linear combinations of basis elements of degree between $n+m+1$ and $n+m+R_{i}$,

Finally, the almost-grading of $\mathscr{A}$ induces an almost-grading of the current algebra $\overline{\mathfrak{g}}$ by setting $\overline{\mathfrak{g}}_{n}=\mathfrak{g} \otimes \mathscr{A}_{n}$. We obtain

$$
\overline{\mathfrak{g}}=\bigoplus_{n \in \mathbb{Z}} \overline{\mathfrak{g}}_{n}, \quad \operatorname{dim} \overline{\mathfrak{g}}_{n}=K \cdot \operatorname{dim} \mathfrak{g} .
$$

4.5. Triangular Decomposition and Filtrations. Let $\mathscr{U}$ be one of the above introduced algebras (including the current algebra). On the basis of the almost-grading we obtain a triangular decomposition of the algebras

$$
\mathscr{U}=\mathscr{U}_{[+]} \oplus \mathscr{U}_{[0]} \oplus \mathscr{U}_{[-]},
$$

where

$$
\mathscr{U}_{[+]}:=\bigoplus_{m>0} \mathscr{U}_{m}, \quad \mathscr{U}_{[0]}=\bigoplus_{m=-R_{i}}^{m=0} \mathscr{U}_{m}, \quad \mathscr{U}_{[-]}:=\bigoplus_{m<-R_{i}} \mathscr{U}_{m} .
$$

By the almost-gradedness the $[+]$ and $[-]$ subspaces are (infinite dimensional) subalgebras. The $[0]$ spaces in general not. Sometimes we will use critical strip for $\mathscr{U}_{[0]}$. 
With respect to the almost-grading of $\mathscr{F}^{\lambda}$ we can introduce a filtration

$$
\begin{gathered}
\mathscr{F}_{(n)}^{\lambda}:=\bigoplus_{m \geq n} \mathscr{F}_{m}^{\lambda}, \\
\cdots \supseteq \mathscr{F}_{(n-1)}^{\lambda} \supseteq \mathscr{F}_{(n)}^{\lambda} \quad \supseteq \quad \mathscr{F}_{(n+1)}^{\lambda} \quad \cdots
\end{gathered}
$$

Proposition 4.6.

$$
\mathscr{F}_{(n)}^{\lambda}:=\left\{f \in \mathscr{F}^{\lambda} \mid \operatorname{ord}_{P_{i}}(f) \geq n-\lambda, \forall i=1, \ldots, K\right\} .
$$

This proposition is very important. In case that $O$ has more than one point there are certain choices, e.g. numbering of the points in $O$, different rules, etc. involved in defining the almost-grading. Hence, if the choices are made differently the subspaces $\mathscr{F}_{n}^{\lambda}$ might depend on them, and consequently also the almost-grading. But by this proposition the induced filtration is indeed canonically defined via the splitting of $A$ into $I$ and $O$.

Moreover, different choices will give equivalent almost-grading. We stress the fact, that under a KN algebra we will always understand one of introduced the algebras (or even some others still to come) together with an almost-grading (resp. equivalence class of almost-grading) introduced by the splitting $A=I \cup O$.

\section{Central Extensions}

Central extension of our algebras appear naturally in the context of quantization and regularization of actions. In Section 6.1 we will see a typical example. Of course they are also of independent mathematical interest.

5.1. Central Extensions and Cocycles. A central extension of a Lie algebra $W$ is a special Lie algebra structure on the vector space direct sum $\widehat{W}=\mathbb{C} \oplus W$. If we denote $\hat{x}:=(0, x)$ and $t:=(1,0)$ then the Lie structure is given by

$$
[\hat{x}, \hat{y}]=\widehat{[x, y]}+\psi(x, y) \cdot t, \quad[t, \widehat{W}]=0, \quad x, y \in W .
$$

The map $x \mapsto \hat{x}=(0, x)$ is a linear splitting map. $\widehat{W}$ will be a Lie algebra, e.g. will fulfill the Jacobi identity, if and only if $\psi$ is antisymmetric and fulfills the Lie algebra 2-cocycle condition

$$
0=d_{2} \psi(x, y, z):=\psi([x, y], z)+\psi([y, z], x)+\psi([z, x], y) .
$$

A 2-cochain $\psi$ is a coboundary if there exists a $\varphi: W \rightarrow \mathbb{C}$ such that

$$
\psi(x, y)=\varphi([x, y]) .
$$

One easily shows that a coboundary is a cocycle. Hence, we can define the second Lie algebra cohomology $\mathrm{H}^{2}(W, \mathbb{C})$ of $W$ with values in the trivial module $\mathbb{C}$ as this quotient.

There is the notion of equivalence of central extensions. For the definition in terms of short exact sequences, I refer to the standard text books. Equivalently, they 
can be described by a different choice of the linear splitting map. Instead of $x \mapsto \hat{x}=(0, x)$ one chooses $x \mapsto \hat{x}^{\prime}=(\varphi, x)$ with $\varphi: W \rightarrow \mathbb{C}$ a linear form. For $\varphi \neq 0$ the Lie structure corresponding to (54) will be different, but equivalent. In fact in (54) we obtain a different 2-cocycle $\psi^{\prime}$.

From this definition follows that that two central extensions are equivalent if and only if the difference of their defining 2-cocycles $\psi$ and $\psi^{\prime}$ is a coboundary. In this way the second Lie algebra cohomology $\mathrm{H}^{2}(W, \mathbb{C})$ classifies equivalence classes of central extensions. The class $[0]$ corresponds to the trivial central extension. In this case the splitting map is a Lie homomorphism. To construct central extensions of our algebras we have to find such Lie algebra 2-cocycles.

Clearly, equivalent central extensions are isomorphic. The opposite is not true. Furthermore, in our case we can always rescale the central element by multiplying it with a nonzero scalar. This is not an equivalence of central extensions but nevertheless an irrelevant modification. Hence we will mainly be interested in central extensions modulo equivalence and rescaling. They are classified by $[0]$ and the elements of the projectivized cohomology space $\mathbb{P}\left(\mathrm{H}^{2}(W, \mathbb{C})\right.$ ).

In the classical case we have $\operatorname{dim} \mathrm{H}^{2}(\mathscr{W}, \mathbb{C})=1$, hence there are only two essentially different central extensions, the splitting one given by the direct sum $\mathbb{C} \oplus \mathscr{W}$ of Lie algebras and the up to equivalence and rescaling unique non-trivial one, the Virasoro algebra $\mathscr{V}$.

Remark 5.1. Given a vector space bases $\left\{e_{\rho} \mid \rho \in \mathscr{R}\right\}$ of $W$, a vector space basis of $\widehat{W}$ will be given by $\left\{\hat{e}_{\rho}:=\left(0, e_{\rho}\right) \mid \rho \in \mathscr{R}, t:=(1,0)\right\}$. An equivalent central extension can be described as a change of basis and rescaling of the form

$$
\hat{e}_{\rho}^{\prime}=\hat{e}_{\rho}+\varphi\left(e_{\rho}\right) t, \quad t^{\prime}=\alpha \cdot t, \alpha \in \mathbb{C}^{*} .
$$

5.2. Geometric Cocycles. The defining cocycle for the Virasoro algebra obviously does not make any sense in the higher genus and/or multi-point case. We need a geometric description. For this we have first to introduce connections.

5.2.1. Projective and Affine Connections. Let $\left(U_{\alpha}, z_{\alpha}\right)_{\alpha \in J}$ be a covering of the Riemann surface $\Sigma$ by holomorphic coordinates with transition functions $z_{\beta}=f_{\beta \alpha}\left(z_{\alpha}\right)$.

Definition 5.2. (a) A system of local (holomorphic, meromorphic) functions $R=$ $\left(R_{\alpha}\left(z_{\alpha}\right)\right)$ is called a (holomorphic, meromorphic) projective connection if it transforms as

$$
R_{\beta}\left(z_{\beta}\right) \cdot\left(f_{\beta, \alpha}^{\prime}\right)^{2}=R_{\alpha}\left(z_{\alpha}\right)+S\left(f_{\beta, \alpha}\right), \quad \text { with } \quad S(h)=\frac{h^{\prime \prime \prime}}{h^{\prime}}-\frac{3}{2}\left(\frac{h^{\prime \prime}}{h^{\prime}}\right)^{2},
$$

the Schwartzian derivative. Here ' denotes differentiation with respect to the coordinate $z_{\alpha}$. 
(b) A system of local (holomorphic, meromorphic) functions $T=\left(T_{\alpha}\left(z_{\alpha}\right)\right)$ is called a (holomorphic, meromorphic) affine connection if it transforms as

$$
T_{\beta}\left(z_{\beta}\right) \cdot\left(f_{\beta, \alpha}^{\prime}\right)=T_{\alpha}\left(z_{\alpha}\right)+\frac{f_{\beta, \alpha}^{\prime \prime}}{f_{\beta, \alpha}^{\prime}} .
$$

Every Riemann surface admits a holomorphic projective connection [20], [19]. Given a point $P$ then there exists always a meromorphic affine connection holomorphic outside of $P$ and having maximally a pole of order one there [40].

From their very definition it follows that the difference of two affine (projective) connections will be a (quadratic) differential. Hence, after fixing one affine (projective) connection all others are obtained by adding (quadratic) differentials.

5.2.2. The Function Algebra $\mathscr{A}$. We consider $\mathscr{A}$ as an abelian Lie algebra. Let $C$ be an arbitrary smooth but not necessarily connected curve. We set

$$
\psi_{C}^{1}(g, h):=\frac{1}{2 \pi \mathrm{i}} \int_{C} g d h, \quad g, h \in \mathscr{A} .
$$

5.2.3. The Current Algebra $\overline{\mathfrak{g}}$. For $\overline{\mathfrak{g}}=\mathfrak{g} \otimes \mathscr{A}$ we first have to fix $\beta$ a symmetric, invariant, bilinear form on $\mathfrak{g}$ (not necessarily non-degenerate). Invariance means that we have $\beta([x, y], z)=\beta(x,[y, z])$ for all $x, y, z \in \mathfrak{g}$. The cocycle is given as

$$
\psi_{C, \beta}^{2}(x \otimes g, y \otimes h):=\beta(x, y) \cdot \frac{1}{2 \pi \mathrm{i}} \int_{C} g d h, \quad x, y \in \mathfrak{g}, g, h \in \mathscr{A} .
$$

5.2.4. The Vector Field Algebra $\mathscr{L}$. Here it is a little bit more delicate. First we have to choose a (holomorphic) projective connection $R$. We define

$$
\psi_{C, R}^{3}(e, f):=\frac{1}{24 \pi \mathrm{i}} \int_{C}\left(\frac{1}{2}\left(e^{\prime \prime \prime} f-e f^{\prime \prime \prime}\right)-R \cdot\left(e^{\prime} f-e f^{\prime}\right)\right) d z
$$

Only by the term related with the projective connection it will be a well-defined differential, i.e. independent of the coordinate chosen. It is shown in [40] that it is a cocycle. Another choice of a projective connection will result in a cohomologous one. Hence, the equivalence class of the central extension will be the same.

5.2.5. The Differential Operator Algebra $\mathscr{D}^{1}$. For the differential operator algebra the cocycles for $\mathscr{A}$ can be extended by zero on the subspace $\mathscr{L}$. The cocycles for $\mathscr{L}$ can be pulled back. In addition there is a third type of cocycles mixing $\mathscr{A}$ and $\mathscr{L}$ :

$$
\psi_{C, T}^{4}(e, g):=\frac{1}{24 \pi \mathrm{i}} \int_{C}\left(e g^{\prime \prime}+T e g^{\prime}\right) d z, \quad e \in \mathscr{L}, g \in \mathscr{A}
$$

with an affine connection $T$, with at most a pole of order one at a fixed point in $O$. Again, a different choice of the connection will not change the equivalence class. For more details on the cocycles see [46]. 
5.2.6. The Lie Superalgebra $\mathscr{S}$. Here we have to take into account that it is not a Lie algebra. Hence, the Jacobi identity has to be replaced by the super-Jacobi identity. The conditions for being a cocycle for the superalgebra cohomology will change too. Recall the definition of the algebra from Section 3.6, in particular that the even elements (parity 0) are the vector fields and the odd elements (parity 1) are the half-forms. A bilinear form $c$ is a cocycle if the following is true. The bilinear map $c$ will be symmetric if $x$ and $y$ are odd, otherwise it will be antisymmetric.

$$
c(x, y)=-(-1)^{\bar{x} \bar{y}} c(x, y) .
$$

The super-cocycle condition reads as

$$
(-1)^{\bar{x} \bar{z}} c(x,[y, z])+(-1)^{\bar{y} \bar{x}} c(y,[z, x])+(-1)^{\bar{z} \bar{y}} c(z,[x, y])=0 .
$$

With the help of $c$ we can define central extensions in the Lie superalgebra sense. If we put the condition that the central element is even then the cocycle $c$ has to be an even map and $c$ vanishes for pairs of elements of different parity.

By convention we denote vector fields by $e, f, g, \ldots$ and $-1 / 2$-forms by $\varphi, \psi, \chi, .$. and get

$$
c(e, \varphi)=0, \quad e \in \mathscr{L}, \varphi \in \mathscr{F}^{-1 / 2} .
$$

The super-cocycle conditions for the even elements is just the cocycle condition for the Lie subalgebra $\mathscr{L}$. The only other nonvanishing super-cocycle condition is for the (even,odd,odd) elements and reads as

$$
c(e,[\varphi, \psi])-c(\varphi, e . \psi)-c(\psi, e . \varphi)=0 .
$$

Here the definition of the product $[e, \psi]:=e . \psi$ was used.

If we have a cocycle $c$ for the algebra $\mathscr{S}$ we obtain by restriction a cocycle for the algebra $\mathscr{L}$. For the mixing term we know that $c(e, \psi)=0$. A naive try to put just anything for $c(\varphi, \psi)$ (for example 0) will not work as (67) relates the restriction of the cocycle on $\mathscr{L}$ with its values on $\mathscr{F}^{-1 / 2}$.

Proposition 5.3. [52] Let $C$ be any closed (differentiable) curve on $\Sigma$ not meeting the points in $A$, and let $R$ be any (holomorphic) projective connection then the bilinear extension of

$$
\begin{aligned}
\Phi_{C, R}(e, f) & :=\frac{1}{24 \pi \mathrm{i}} \int_{C}\left(\frac{1}{2}\left(e^{\prime \prime \prime} f-e f^{\prime \prime \prime}\right)-R \cdot\left(e^{\prime} f-e f^{\prime}\right)\right) d z \\
\Phi_{C, R}(\varphi, \psi) & :=-\frac{1}{24 \pi \mathrm{i}} \int_{C}\left(\varphi^{\prime \prime} \cdot \psi+\varphi \cdot \psi^{\prime \prime}-R \cdot \varphi \cdot \psi\right) d z \\
\Phi_{C, R}(e, \varphi) & :=0
\end{aligned}
$$

gives a Lie superalgebra cocycle for $\mathscr{S}$, hence defines a central extension of $\mathscr{S}$. A different projective connection will yield a cohomologous cocycle. 
A similar formula was given by Bryant in [10]. By adding the projective connection in the second part of (68) he corrected some formula appearing in [3]. He only considered the two-point case and only the integration over a separating cycle. See also [24] for the multi-point case, where still only the integration over a separating cycle is considered.

In contrast to the differential operator algebra case the two parts cannot be prescribed independently. Only with the same integration path (more precisely, homology class) and the given factors in front of the integral it will work. The reason for this that (67) relates both.

5.3. Uniqueness and Classification of Central Extensions. Our cocycles depend on the choice of the connections $R$ and $T$. But different choices will not change the equivalence class. Hence, this ambiguity does not disturb us. What really matters is that they depend on the integration curve $C$ chosen.

In contrast to the classical situation, for the higher genus and/or multi-point situation there are many essentially different closed curves and hence many nonequivalent central extensions defined by the integration.

But we should take into account that we want to extend the almost-grading from our algebras to the centrally extended ones. This means we take $\operatorname{deg} \hat{x}:=\operatorname{deg} x$ and assign a degree $\operatorname{deg}(t)$ to the central element $t$, and still obtain almost-gradedness.

This is possible if and only if our defining cocycle $\psi$ is "local" in the following sense (the name was introduced in the two point case by Krichever and Novikov in [26]). There exists $M_{1}, M_{2} \in \mathbb{Z}$ such that

$$
\forall n, m: \quad \psi\left(W_{n}, W_{m}\right) \neq 0 \Longrightarrow M_{1} \leq n+m \leq M_{2} .
$$

Here $W$ stands for any of our algebras (including the supercase). Very important, "local" is defined in terms of the almost-grading, and the grading itself depends on the splitting $A=I \cup O$. Hence what is "local" depends on the splitting too.

We will call a cocycle bounded (from above) if there exists $M \in \mathbb{Z}$ such that

$$
\forall n, m: \quad \psi\left(W_{n}, W_{m}\right) \neq 0 \Longrightarrow n+m \leq M .
$$

Similarly bounded from below is defined. Locality means bounded from above and below.

Given a cocycle class we call it bounded (resp. local) if and only if it contains a representing cocycle which is bounded (resp. local). Not all cocycles in a bounded class have to be bounded. If we choose as integration path a separating cocycle $C_{S}$, or one of the $C_{i}$ then the above introduced geometric cocycles are local, resp. bounded. Recall that in this case integration can be done by calculating residues at the in-points or at the out-points. All these cocycles are cohomologically nontrivial. The theorems in the following concern the opposite direction. They were treated in my works [46], [47], [52].

I start with the vector field case as it will give a model for all the results. 
Theorem 5.4. [46] Let $\mathscr{L}$ be the Krichever-Novikov vector field algebra.

(a) The space of bounded cohomology classes is $K$-dimensional $(K=\# I)$. A basis is given by setting the integration path in (62) to $C_{i}, i=1, \ldots, K$ the little (deformed) circles around the points $P_{i} \in I$.

(b) The space of local cohomology classes is one-dimensional. A generator is given by integrating (62) over a separating cocycle $C_{S}$.

(c) Up to equivalence and rescaling there is only one non-trivial one-dimensional central extension of the vector field algebra $\mathscr{L}$ which allows an extension of the almost-grading.

Part (c) says that the result is completely analogous to the case of the Witt algebra. Here I like to repeat again the fact that for $\mathscr{L}$ depending on the set $A$ and its possible splittings into two disjoint subsets there are different almost-gradings. Hence, the "unique" central extension finally obtained will also depend on the splitting. Only in the two point case there is only one splitting possible.

The above theorem is a model for all other classification results. We will always obtain a statement about the bounded (from above) cocycles and then for the local cocycles.

As $\mathscr{A}$ is an abelian Lie algebra every skew-symmetric bilinear form will be a nontrivial cocycle. Hence, there is no hope of uniqueness. But if we add the condition of $\mathscr{L}$-invariance, i.e.

$$
\psi(e . g, h)+\psi(g, e . h)=0, \quad \forall e \in \mathscr{L}, g, h \in \mathscr{A}
$$

things will change.

Let us denote the the subspace of local cohomology classes by $\mathrm{H}_{l o c}^{2}$, and the subspace of local and $\mathscr{L}$-invariant by $\mathrm{H}_{\mathscr{L}, l o c}^{2}$. Note that the condition is only required for at least one representative in the cohomology class. We collect a part of the results for the other algebras in the following theorem.

\section{Theorem 5.5.}

(1) $\operatorname{dim} \mathrm{H}_{\mathscr{L}, l o c}^{2}(\mathscr{A}, \mathbb{C})=1$,

(2) $\operatorname{dim}_{\text {loc }}^{2}(\mathscr{S}, \mathbb{C})=1$,

(3) $\operatorname{dim} \mathrm{H}_{\text {loc }}^{2}\left(\mathscr{D}^{1}, \mathbb{C}\right)=3$,

(4) $\operatorname{dim} \mathrm{H}_{\text {loc }}^{2}(\overline{\mathfrak{g}}, \mathbb{C})=1$ for $\mathfrak{g}$ a simple finite-dimensional Lie algebra,

A basis of the cohomology spaces are given by taking the cohomology classes of the cocycles (60), (61), (62), (63), (68) obtained by integration over a separating cycle $C_{S}$.

Correspondingly, we obtain also for these algebras the corresponding result about uniqueness of almost-graded central extensions. For the differential operator algebra we got three independent cocycles. This generalizes results of [1] for the classical case. 
For the bounded cocycle classes we have to multiply the dimensions above by $K$. For the supercase with odd central elements the bounded cohomology vanishes.

For $\mathfrak{g}$ a reductive Lie algebra and the cocycle $\mathscr{L}$-invariant if restricted to the abelian part, a complete classification of local cocycle classes for $\overline{\mathfrak{g}}$ can be found in [47. Note that in the case of a simple Lie algebra every symmetric, invariant bilinear form $\beta$ is a multiple of the Cartan-Killing form.

I like to mention that in all the applications I know of, the cocycles coming from representations, regularizations, etc. are local. Hence, the uniqueness or classification can be used.

\section{Further Results}

Above the basic concepts, results about the structure of these Krichever-Novikov type algebras and their central extensions were treated. Of course, this does not close the story. I will add some further important constructions and applications but due to space limitations only in a very condensed manner.

\subsection{Semi-Infinite Forms and Fermionic Fock Space Representations.}

Our Krichever-Novikov vector field algebras $\mathscr{L}$ have as Lie modules the spaces $\mathscr{F}^{\lambda}$. These representations are not of the type physicists are usually interested in, as there is no ground state (no vacuum). There are neither creation nor annihilation operators which can be used to construct the full representation out of a vacuum state.

To obtain such desired representations the almost-grading comes into play. First, using the grading of $\mathscr{F}^{\lambda}$ it is possible to construct starting from $\mathscr{F}^{\lambda}$, the forms of weight $\lambda \in 1 / 2 \mathbb{Z}$, the semi-infinite wedge forms $\mathscr{H}^{\lambda}$.

The vector space $\mathscr{H}^{\lambda}$ is generated by basis elements which are formal expressions

$$
\Phi=f_{\left(i_{1}\right)}^{\lambda} \wedge f_{\left(i_{2}\right)}^{\lambda} \wedge f_{\left(i_{3}\right)}^{\lambda} \wedge \cdots
$$

where $\left(i_{1}\right)=\left(m_{1}, p_{1}\right)$ is a double index indexing our basis elements. The indices are in strictly increasing lexicographical order. They are stabilizing in the sense that they will increase exactly by one starting from a certain point, depending on $\Phi$. The action of $\mathscr{L}$ can be extended by Leibniz rule from $\mathscr{F}^{\lambda}$ to $\mathscr{H}^{\lambda}$. But a problem arises. For elements of the critical strip $\mathscr{L}_{[0]}$ of the algebra $\mathscr{L}$ it might happen that it produces infinitely many contributions. The action has to be regularized (as physicists like to call it, but it is a well-defined mathematical procedure).

Here the almost-grading has his second appearance. By the (strong) almostgraded module structure of $\mathscr{F}^{\lambda}$ the algebra $\mathscr{L}$ can be imbedded into the Lie algebra of both-sided infinite matrices

$$
\overline{g l}(\infty):=\left\{A=\left(a_{i j}\right)_{i, j \in \mathbb{Z}} \mid \exists r=r(A), \text { such that } a_{i j}=0 \text { if }|i-j|>r\right\},
$$


with "infinitely many diagonals". The embedding will depend on the weight $\lambda$. For $\overline{g l}(\infty)$ there exists a procedure for the regularization of the action on the semi-infinite wedge forms [12], [22], see also [23] for a nice pedagogical treatment. In particular, there is a unique non-trivial central extension $\widehat{g l}(\infty)$. If we pull-back the defining cocycle for the extension we obtain a central extension $\widehat{\mathscr{L}_{\lambda}}$ of $\mathscr{L}$ and the required regularization of the action of $\widehat{\mathscr{L}_{\lambda}}$ on $\mathscr{H}^{\lambda}$. As the embedding of $\mathscr{L}$ depends on the weight $\lambda$ the cocycle will do so. The pull-back cocycle will be local. Hence, by the classification results of Section 5.3 it is the unique central extension class defined by (62) integrated over $C_{S}$ (up to a $\lambda$ dependent rescaling).

In $\mathscr{H}^{\lambda}$ there are invariant subspaces, which are generated by a certain "vacuum vectors". Such a vacuum $\Phi_{T}$ is given by an element of the form (72) which starts with the element $f_{(T, 1)}^{\lambda}$, and the indices for the following ones increase always by one. The subalgebra $\mathscr{L}_{[+]}$annihilates the vacuum, the central element and the other elements of degree zero act by multiplication with a constant on the vacuum and the whole representation state is generated by $\mathscr{L}_{[-]} \oplus \mathscr{L}_{[0]}$ from the vacuum.

As the function algebra $\mathscr{A}$ operates as multiplication operators on $\mathscr{F}^{\lambda}$ the above representation can be extended to the algebra $\mathscr{D}^{1}$ (see details in [40]) after one passes over to central extensions. The cocycle again is local and hence, up to coboundary, it will be a certain linear combination of the 3 generating cocycles for the differential operator algebra. In fact it will be

$$
c_{\lambda}\left[\psi_{C_{S}}^{3}\right]+\frac{2 \lambda-1}{2}\left[\psi_{C_{S}}^{4}\right]-\left[\psi_{C_{S}}^{1}\right], \quad c_{\lambda}:=-2\left(6 \lambda^{2}-6 \lambda+1\right) .
$$

Recall that $\psi^{3}$ is the cocycle for the vector field algebra, $\psi^{1}$ the cocycle for the function algebra, and $\psi^{4}$ the mixing cocycle, see [46] for details. Note that the expression for $c_{\lambda}$ appears also in Mumford's formula [36] relating divisors on the moduli space of curves.

Also the representation on $\mathscr{H}^{\lambda}$ gives a projective representation of the algebra of $\mathscr{D}_{\lambda}$ of differential operators of all orders. It is exactly the combination (74) which lifts to a cocycle for $\mathscr{D}_{\lambda}$ and gives a central extension $\widehat{\mathscr{D}}_{\lambda}$. For $\mathscr{L}$ we could rescale the central element. Hence the central extension $\widehat{\mathscr{L}}$ did not depend essentially on the weight. Here this is different. The central extension $\widehat{\mathscr{D}^{1}} \lambda$ depends on it.

For the centrally extended current algebras $\widehat{\mathfrak{g}}$, the affine algebra of KN type, in a similar way fermionic Fock space representations can be constructed, see [63], [55].

6.2. $b-c$ Systems. Related to the above there are other quantum algebra systems which can be realized on $\mathscr{H}^{\lambda}$. On the space $\mathscr{H}^{\lambda}$ the forms $\mathscr{F}^{\lambda}$ act by wedging elements $f^{\lambda} \in \mathscr{F}^{\lambda}$ in front of the semi-infinite wedge form, i.e.

$$
\Phi \mapsto f^{\lambda} \wedge \Phi .
$$


Using the Krichever-Novikov duality pairing (34) to contract in the semi-infinite wedge form the entries with the form $f^{1-\lambda} \in \mathscr{F}^{1-\lambda}$ the latter form will act $\mathscr{H}^{\lambda}$. For $\Phi$ a basis element (72) of $\mathscr{H}^{\lambda}$ the contraction is defines via

$$
i\left(f^{1-\lambda}\right) \Phi=\sum_{l=1}^{\infty}(-1)^{l-1}\left\langle f^{1-\lambda}, f_{i_{l}}^{\lambda}\right\rangle \cdot f_{\left(i_{1}\right)}^{\lambda} \wedge f_{\left(i_{2}\right)}^{\lambda} \wedge \cdots \breve{f}_{\left(i_{l}\right)}^{\lambda} \cdots
$$

Here $\breve{f}_{\left(i_{l}\right)}^{\lambda}$ indicates as usual that this element will not be there anymore.

Both operations together create a Clifford algebra structure, which is sometimes called a $b-c$ system, see [40], [43], [53].

6.3. Sugawara Representation. Given an admissible representation of the centrally extended current algebra $\widehat{\mathfrak{g}}$ we can construct the so called Sugawara operators. Here admissible means, that the central element operates as constant $\times$ identity, and that every element $v$ in the representation space will be annihilated by the elements in $\widehat{\mathfrak{g}}$ of sufficiently high degree (the degree depends on the element $v$ ). The Sugawara operator is an infinite formal sum of operators and is constructed as the product of the current operators which are again formal infinite sum of operators. To make the product well-defined a normal ordering has to be set, which moves the annihilation operators to the right to act first. It turns out that after some rescaling the operators appearing in the formal sum of the Sugawara operators give a representation of a centrally extended vector field algebra $\mathscr{L}$. The central extension is due to the appearance of the normal ordering. Again the defining cocycle is local and we know that the central extension defined by the representation is the central extension given by our geometric cocycle $\psi_{C_{S}}^{3}$. See [54], [44], [43] for details.

\subsection{Wess-Zumino-Novikov-Witten models and Knizhnik-Zamolodchikov Con-} nection. Despite the fact, that it is a very important application, the following description is extremely condensed. More can be found in [55], [56], [45]. See also [66], [53]. Wess-Zumino-Novikov-Witten (WZNW) models are defined on the basis of a fixed finite-dimensional Lie algebra $\mathfrak{g}$. One considers families of representations of the affine $\mathrm{KN}$ algebras $\widehat{\mathfrak{g}}$ (which is an almost-graded central extension of the current algebra $\overline{\mathfrak{g}}$ of KN type) defined over the moduli space of Riemann surfaces of genus $g$ with $K+1$ marked points and splitting of type $(K, 1)$. The single point in $O$ will be a reference point. The data of the moduli of the Riemann surface and the marked points enter the definition of the algebra $\widehat{\mathfrak{g}}$ and the representation. The construction of certain co-invariants yields a special vector bundle of finite rank over moduli space, called the vector bundle of conformal blocks. With the help of the Krichever Novikov vector field algebra, and using the Sugawara construction, the Knizhnik-Zamolodchikov (KZ) connection is given. It is projectively flat. An essential fact is that certain elements in the critical strip $\mathscr{L}_{[0]}$ of the vector field algebra correspond to infinitesimal deformations of the moduli and to moving the marked 
points. This gives a global operator approach in contrast to the semi-local approach of Tsuchiya, Ueno, and Yamada [67].

6.5. Geometric Deformations of the Witt and Virasoro Algebra. As the second Lie algebra cohomology of the Witt and Virasoro algebra with values in their adjoint module vanishes [15], [51], [14] both are formally and infinitesimally rigid. This means that all formal (and infinitesimal) families where the special fiber is one of these algebras are equivalent to the trivial one. Nevertheless, we showed in [15] that there exists naturally defined families of Krichever-Novikov vector field algebras defined for the torus with two marked points [41], [11], [34]. These families are obtained by a geometric degeneration process. The families have as special element the Witt algebra (resp. Virasoro algebra). All other fibers are non-isomorphic to it. Hence, these families are even locally non-trivial. This is a phenomena which can only be observed for infinite dimensional algebras. See also the case of affine Lie algebra and some general treatment in [16], [17], [49].

\section{LAX Operator Algebras}

Recently, a new class of current type algebras appeared, the Lax operator algebras. As the naming indicates, they are related to infinite dimensional integrable systems [65]. The algebras were introduced by Krichever [25], and Krichever and Sheinman [29]. Here I will report on their definition.

Compared to the KN current type algebra we will allow additional singularities which will play a special role. The points where these singularities are allowed are called weak singular points. The set is denoted by

$$
W=\left\{\gamma_{s} \in \Sigma \backslash A \mid s=1, \ldots, R\right\} .
$$

Let $\mathfrak{g}$ be one of the classical matrix algebras $\mathfrak{g l}(n), \mathfrak{s l}(n), \mathfrak{s o}(n), \mathfrak{s p}(2 n)$. We assign to every point $\gamma_{s}$ a vector $\alpha_{s} \in \mathbb{C}^{n}$ (resp. $\in \mathbb{C}^{2 n}$ for $\mathfrak{s p}(2 n)$ ). The system

$$
\mathscr{T}:=\left\{\left(\gamma_{s}, \alpha_{s}\right) \in \Sigma \times \mathbb{C}^{n} \mid s=1, \ldots, R\right\}
$$

is called Tyurin data.

Remark 7.1. In case that $R=n \cdot g$ and for generic values of $\left(\gamma_{s}, \alpha_{s}\right)$ with $\alpha_{s} \neq 0$ the tuples of pairs $\left(\gamma_{s},\left[\alpha_{s}\right]\right)$ with $\left[\alpha_{s}\right] \in \mathbb{P}^{n-1}(\mathbb{C})$ parameterize semi-stable rank $n$ and degree $n g$ framed holomorphic vector bundles as shown by Tyurin [68]. Hence, the name Tyurin data.

We consider $\mathfrak{g}$-valued meromorphic functions

$$
L: \Sigma \rightarrow \mathfrak{g},
$$

which are holomorphic outside $W \cup A$, have at most poles of order one (resp. of order two for $\mathfrak{s p}(2 n)$ ) at the points in $W$, and fulfill certain conditions at $W$ depending on $\mathscr{T}$. To describe them let us fix local coordinates $w_{s}$ centered at $\gamma_{s}, s=1, \ldots, R$. 
For $\mathfrak{g l}(n)$ the conditions are as follows. For $s=1, \ldots, R$ we require that there exist $\beta_{s} \in \mathbb{C}^{n}$ and $\kappa_{s} \in \mathbb{C}$ such that the function $L$ has the following expansion at $\gamma_{s} \in W$

$$
L\left(w_{s}\right)=\frac{L_{s,-1}}{w_{s}}+L_{s, 0}+\sum_{k>0} L_{s, k} w_{s}^{k},
$$

with

$$
L_{s,-1}=\alpha_{s} \beta_{s}^{t}, \quad \operatorname{tr}\left(L_{s,-1}\right)=\beta_{s}^{t} \alpha_{s}=0, \quad L_{s, 0} \alpha_{s}=\kappa_{s} \alpha_{s} .
$$

In particular, if $L_{s,-1}$ is non-vanishing then it is a rank 1 matrix, and if $\alpha_{s} \neq 0$ then it is an eigenvector of $L_{s, 0}$. The requirements (81) are independent of the chosen coordinates $w_{s}$.

It is not at all clear that the commutator of two such matrix functions fulfills again these conditions. But it is shown in [29] that they indeed close to a Lie algebra (in fact in the case of $\mathfrak{g l}(n)$ they constitute an associative algebra under the matrix product). If one of the $\alpha_{s}=0$ then the conditions at the point $\gamma_{s}$ correspond to the fact, that $L$ has to be holomorphic there. If all $\alpha_{s}$ 's are zero or $W=\emptyset$ we obtain back the current algebra of KN type. In some sense the Lax operator algebras generalize them. But their definition is restricted to the case that our finite-dimensional Lie algebra has to be one from the list. In the bundle interpretation of the Tyurin data the $\mathrm{KN}$ case corresponds to the trivial rank $n$ bundle.

For $\mathfrak{s l}(n)$ the only additional condition is that in (80) all matrices $L_{s, k}$ are traceless. The conditions (81) remain unchanged.

In the case of $\mathfrak{s o}(n)$ one requires that all $L_{s, k}$ in (80) are skew-symmetric. In particular, they are trace-less. Following [29] the set-up has to be slightly modified. First only those Tyurin parameters $\alpha_{s}$ are allowed which satisfy $\alpha_{s}^{t} \alpha_{s}=0$. Then the 1. relation in (81) is changed to obtain

$$
L_{s,-1}=\alpha_{s} \beta_{s}^{t}-\beta_{s} \alpha_{s}^{t}, \quad \operatorname{tr}\left(L_{s,-1}\right)=\beta_{s}^{t} \alpha_{s}=0, \quad L_{s, 0} \alpha_{s}=\kappa_{s} \alpha_{s} .
$$

For $\mathfrak{s p}(2 n)$ we consider a symplectic form $\hat{\sigma}$ for $\mathbb{C}^{2 n}$ given by a non-degenerate skew-symmetric matrix $\sigma$. The Lie algebra $\mathfrak{s p}(2 n)$ is the Lie algebra of matrices $X$ such that $X^{t} \sigma+\sigma X=0$. The condition $\operatorname{tr}(X)=0$ will be automatic. At the weak singularities we have the expansion

$$
L\left(w_{s}\right)=\frac{L_{s,-2}}{w_{s}^{2}}+\frac{L_{s,-1}}{w_{s}}+L_{s, 0}+L_{s, 1} w_{s}+\sum_{k>1} L_{s, k} w_{s}^{k} .
$$

The condition (81) is modified as follows (see [29]): there exist $\beta_{s} \in \mathbb{C}^{2 n}, v_{s}, \kappa_{s} \in \mathbb{C}$ such that

$$
L_{s,-2}=v_{s} \alpha_{s} \alpha_{s}^{t} \sigma, \quad L_{s,-1}=\left(\alpha_{s} \beta_{s}^{t}+\beta_{s} \alpha_{s}^{t}\right) \sigma, \quad \beta_{s}{ }^{t} \sigma \alpha_{s}=0, \quad L_{s, 0} \alpha_{s}=\kappa_{s} \alpha_{s} .
$$

Moreover, we require $\alpha_{s}^{t} \sigma L_{s, 1} \alpha_{s}=0$. Again under the point-wise matrix commutator the set of such maps constitute a Lie algebra. 
The next step is to introduce an almost-graded structure for these Lax operator algebras induced by a splitting of the set $A=I \cup O$. This is done for the twopoint case in [29] and for the multi-point case in [58]. From the applications there is again a need to classify almost-graded central extensions. The author obtained this jointly with O. Sheinman in [57], [58], see also [50] for an overview. For the Lax operator algebras associated to the simple algebras $\mathfrak{s l}(n), \mathfrak{s o}(n), \mathfrak{s p}(n)$ it will be unique (meaning: given a splitting of $A$ there is an almost-grading and with respect to this there is up to equivalence and rescaling only one non-trivial almost-graded central extension). For $\mathfrak{g l}(n)$ we obtain two independent local cocycle classes if we assume $\mathscr{L}$-invariance on the reductive part.

\section{SOME HistORICAL REMARKS}

In this section I will give some historical remarks (also on related works) on Krichever-Novikov type algebras. Space limitations do not allow to give a complete list of references. For this I have to refer to [53].

In 1987 the ground-breaking work of Krichever and Novikov [26], [27], [28] in the two-point case initiated the subject. They introduced the vector field algebra, the function algebra and the affine algebra with their almost-graded structure. To acknowledge their work these algebras are nowadays called Krichever-Novikov (KN) type algebras. Sheinman joint in by investigating the affine algebras and their representations [59], [60], [61], [62], [63], [64].

As it should have become clear from this review what should be called a $\mathrm{KN}$ type algebra is not the algebra alone but the algebra together with a chosen almostgrading. It is exactly the step of introducing such an almost-grading which is not straightforward.

From the application in CFT, string theory, etc. there was clearly the need to pass over to a multi-point picture. In the multi-point case this was done 1989 by the author in [37], [38], [39], [40]. The almost-grading is induced by splitting of the set $A$ of points where poles are allowed into two non-empty disjoint subsets $I$ and $O$. In the applications quite often such a splitting is naturally given. In the context of fields and strings the points correspond to incoming and outgoing fields and strings respectively. Without considering an almost-grading Dick [13] gave also a generalization of the vector field algebra. He obtained results similar to [37]. Only in the work of Sadov [35] 1990 an almost-grading is also discussed.

Note that in the two-point case there is only one splitting. Hence, quite often one does not mention explicitely the grading for the Witt and Virasoro algebra, respectively the almost-grading for the KN type algebras. Nevertheless, the grading is heavily used.

The genus zero and two-point case is the classical well-studied case. But already for genus zero and more than two points there are interesting things so study, see 
[38], [15], [16], [8], [9], [48]. For genus one, the complex torus case, there is [38], [7], [11], [34], [15], [16].

After the work of Krichever and Novikov appeared physicists got very much interested in these algebras and the possibilities of using these objects for a global operator approach. Especially I like to mention the work of the people around Bonora [3], [4], [5], [6] and by [10]. This includes also the superversions. A lot of more names could be given.

It is not only the possible applications in physics which makes the KN type algebras so interesting. From the mathematical point general infinite dimensional Lie algebras are hard to approach. KN type algebras supply examples of them which are given in a geometrical context, hence (hopefully) better to understand. A typical example of this are the families of geometric deformation of the Witt algebra which I mentioned in Section 6.5 obtained by degenerations of tori. Quite recently also in the context of Jordan Superalgebras and Lie antialgebras [33] examples were constructed on the basis of KN type algebras [30], [31], [24]

In this review we discussed extensively the case of 2nd Lie algebra cohomology with values in the trivial module. But we did not touch the question of the general Lie algebra cohomology of Krichever-Novikov type algebras. Here I refer e.g. to work of Wagemann [69], [70].

\section{REFERENCES}

[1] Arbarello, E., deConcini, C., Kac, V., Procesi, C., Moduli spaces of Curves and representation theory, Comm. Math. Phys. 117 (1988), 1-36

[2] Belavin, A.A., Polyakov, A.M., Zamolodchikov, A.B., Infinite conformal symmetry in twodimensional quantum field theory. Nucl. Phys. B 241, 333-380 (1084)

[3] Bonora, L., Martellini, M., Rinaldi, M., Russo, J., Neveu-Schwarz- and Ramond-type Superalgebras on genus g Riemann surfaces, Phys. Lett. B, 206(3) (1988), 444-450.

[4] Bonora, L., Lugo, A., Matone, M., Russo, J., A global operator formalism on higher genus Riemann surfaces: $b-c$ systems, Comm. Math. Phys. 123 (1989), 329-352.

[5] Bonora, L., Matone, M., Toppan, F., Wu, K., Real weight $b-c$ systems and conformal field theories in higher genus, Nuclear Phys. B 334 (1990), no. 3, 716-744.

[6] Bonora, L., Rinaldi, M., Russo, J., Wu, K., The Sugawara construction on genus g Riemann surfaces, Phys. Lett. B208 (1988), 440-446.

[7] Bremner, M.R., On a Lie algebra of vector fields on a complex torus, J. Math. Phys. 31 (1990), 2033-2034.

[8] Bremner, M.R., Structure of the Lie algebra of polynomial vector fields on the Riemann sphere with three punctures, J. Math. Phys. 32 (1991), 1607-1608

[9] Bremner, M.R., Four-point affine Lie algebras, Proc. Amer. Math. Soc., 123 (1995), 1981-1989.

[10] Bryant, P., Graded Riemann surfaces and Krichever-Novikov algebras, Lett. Math. Phys., 19 (1990), 97-108.

[11] Deck, Th., Deformations from Virasoro to Krichever-Novikov algebras, Phys. Lett. B.251 (1990) $335-540$.

[12] Date, E., Jimbo, M., Miwa, T., Kashiwara, M., Transformation groups for soliton equations, Publ. RIMS, Kyoto 394 (1982) 
[13] Dick, R., Krichever-Novikov-like bases on punctured Riemann surfaces, Lett. Math. Phys. 18 (1989), 255-265

[14] Fialowski, A., Formal rigidity of the Witt and Virasoro Algebra, arXiv:1202.3132

[15] Fialowski, A., Schlichenmaier, M., Global deformations of the Witt algebra of KricheverNovikov type, Comm. Contemp. Math. 5 (6) (2003), 921-946.

[16] Fialowski, A., Schlichenmaier, M., Global geometric deformations of current algebras as Krichever-Novikov type algebras, Comm. Math. Phys. 260 (2005), 579 -612.

[17] Fialowski, A., Schlichenmaier, M., Global Geometric Deformations of the Virasoro algebra, current and affine algebras by Krichever-Novikov type algebras, Inter. Jour. Theor. Phys. Vol. 46, No. 11 (2007) pp.2708 - 2724

[18] Guieu, L., Roger, C.: L'algèbre et le groupe de Virasoro. Les publications CRM, Montreal 2007.

[19] Gunning, R.C., Lectures on Riemann surfaces, Princeton Math. Notees, N.J. 1966.

[20] Hawley, N.S., Schiffer, M., Half-order differentials on Riemann surfaces, Acta Math. 115 (1966), 199-236.

[21] Kac, V.G., Infinite dimensional Lie algebras. Cambridge Univ. Press, Cambridge, 1990.

[22] Kac, V.G., Petersen, D.H. Spin and wedge representations of infinite-dimensional Lie algebras and groups. Proc. Nat. Acad. Sci. USA 78 (1981) 3308-3312.

[23] Kac, V., Raina, A., Highest weight representations of infinite dimensional Lie algebras, World Scientific, 1987.

[24] Kreusch, M., Extensions of superalgebras of Krichever-Novikov type, arXiv:1204.4338v2.

[25] Krichever, I.M., Vector bundles and Lax equations on algebraic curves. Comm. Math. Phys. 229, 229-269 (2002).

[26] Krichever, I.M., Novikov, S.P., Algebras of Virasoro type, Riemann surfaces and structures of the theory of solitons. Funktional Anal. i. Prilozhen. 21, No.2 (1987), 46-63.

[27] Krichever, I.M., Novikov, S.P., Virasoro type algebras, Riemann surfaces and strings in Minkowski space. Funktional Anal. i. Prilozhen. 21, No.4 (1987), 47-61.

[28] Krichever, I.M., Novikov, S.P., Algebras of Virasoro type, energy-momentum tensors and decompositions of operators on Riemann surfaces. Funktional Anal. i. Prilozhen. 23, No.1 (1989), 46-63.

[29] Krichever, I. M., Sheinman, O. K., Lax operator algebras, Funct. Anal. Appl. 41 (2007), 284294.

[30] Leidwanger, S., Morier-Genoud, S., Superalgebras associated to Riemann surfaces: Jordan algebras of Krichever-Novikov type, Int. Math. Res. Notices, doi:10.1093/imrn/rnr 196

[31] Leidwanger, S., Morier-Genoud, S., Universal enveloping algebras of Lie antialgebras, Algebras and Representation Theory 15(1) (2012), 1-27

[32] Moody, R. V. Euclidean Lie algebras, Canad. J. Math. 21 (1969), 1432 -1454.

[33] Ovsienko, V., Lie antialgebras: prémices, J. of Algebra 325 (1) (2011), 216-247

[34] Ruffing, A., Deck, Th., Schlichenmaier, M., String Branchings on complex tori and algebraic representations of generalized Krichever-Novikov algebras, Lett. Math. Phys. 26 (1992), 23-32.

[35] Sadov, V.A. Bases on Multipunctured Riemann Surfaces and Interacting String Amplitudes. Comm. Math. Phys. 136 (1991), 585-597.

[36] Schlichenmaier, M., An Introduction to Riemann Surfaces, Algebraic Curves and Moduli Spaces, 2nd enlarged edition, Springer, 2007. (1st edition published 1989)

[37] Schlichenmaier, M., Krichever-Novikov algebras for more than two points, Lett. Math. Phys. 19(1990), 151-165.

[38] Schlichenmaier, M., Krichever-Novikov algebras for more than two points: explicit generators, Lett. Math. Phys. 19(1990), 327-336.

[39] Schlichenmaier, M., Central extensions and semi-infinite wedge representations of KricheverNovikov algebras for more than two points, Lett. Math. Phys. 20(1990), 33-46. 
[40] Schlichenmaier, M.,Verallgemeinerte Krichever - Novikov Algebren und deren Darstellungen. Ph.D. thesis, Universität Mannheim, 1990.

[41] Schlichenmaier, M., Degenerations of generalized Krichever-Novikov algebras on tori, Journal of Mathematical Physics 34(1993), 3809-3824.

[42] Schlichenmaier, M., Differential operator algebras on compact Riemann surfaces, in: Generalized Symmetries in Physics, Clausthal 1993 (eds. H.-D. Doebner, V.K. Dobrev, A.G. Ushveridze), World Scientific, Singapore, London, 1994, pp.425-434

[43] Schlichenmaier, M., Zwei Anwendungen algebraisch-geometrischer Methoden in der theoretischen Physik: Berezin-Toeplitz-Quantisierung und globale Algebren der zweidimensionalen konformen Feldtheorie”, Habilitation Thesis, University of Mannheim, June 1996.

[44] Schlichenmaier, M., Sugawara operators for higher genus Riemann surfaces, Rep. on Math. Phys. 43(1999), 323-339,

[45] Schlichenmaier, M., Elements of a Global Operator Approach to Wess-Zumino-Novikov-Witten Models, in "Lie theory and its applications in physics III" (July 1999, Clausthal), (eds. H.D. Doebner, V.K.Dobrev, and J. Hilgert), World Scientific, 2000, pp. 204-220. arXiv:math/0001040

[46] Schlichenmaier, M., Local cocycles and central extensions for multi-point algebras of KricheverNovikov type, J. reine angew. Math. 559(2003), 53-94.

[47] Schlichenmaier, M., Higher genus affine Lie algebras of Krichever-Novikov type, Moscow Math. Journal 3(2003), 1395-1427

[48] Schlichenmaier, M., Higher genus affine Lie algebras of Krichever-Novikov type Proceedings of the International Conference on Difference Equations, Special Functions, and Applications, Munich, World-Scientific, 2007.

[49] Schlichenmaier, M., Deformations of the Witt, Virasoro, and Current Algebra, (in) Silvestrov, et. al., Generalized Lie theory in Mathematics, Physics and Beyond, pp. 219-234, Springer 2009

[50] Schlichenmaier, M., Almost-graded central extensions of Lax operator algebras, Banach Center Publ. 93 (2011), 129-144.

[51] Schlichenmaier, M., An elementary proof of the vanishing of the second cohomology of the Witt and Virasoro algebra with values in the adjoint module, arXiv:1111.6624v1, 2011, DOI: 10.1515/forum-2011-0143, February 2012.

[52] Schlichenmaier, M., Lie superalgebras of Krichever-Novikov type. Almost-grading and central extensions, arXiv:1301.0484

[53] Schlichenmaier, M., Krichever-Novikov algebras. Theory and Applications., forthcoming.

[54] Schlichenmaier, M., Sheinman, O. K., Sugawara construction and Casimir operators for Krichever-Novikov algebras, Jour. of Math. Science 92(1998), 3807-3834, translated from Itoki Nauki i Tekhniki, Vol. 38, 1996, q-alg/9512016

[55] Schlichenmaier, M., Sheinman, O.K., Wess-Zumino-Witten-Novikov theory, KnizhnikZamolodchikov equations, and Krichever-Novikov algebras, I.. Russian Math. Surv. (Uspekhi Math. Nauk.) 54 (1999), 213-250, math.QA/9812083

[56] Schlichenmaier, M., Sheinman, O.K., Knizhnik-Zamolodchikov equations for positive genus and Krichever-Novikov algebras, Russian Math. Surv. 59 (2004), No. 4, 737-770,

[57] Schlichenmaier, M., Sheinman, O. K., Central extensions of Lax operator algebras, Uspheki Math. Mauk., 63:4, 2008, 131-172; Russ. Math. Surveys 63:4 (2008), 727-766.

[58] Schlichenmaier, M., Sheinman, O. K., Multipoint Lax operator algebras: almost graded structure and central extension, in preparation

[59] Sheinman, O.K. Elliptic affine Lie algebras. Funktional Anal. i Prilozhen. 24 No.3 (1992), 210219.

[60] Sheinman, O.K., Highest Weight modules over certain quasi-graded Lie algeebras on elliptic curves, Funktional Anal. i Prilozhen. 26, No.3 (1992), 203-208. 
[61] Sheinman, O.K., Affine Lie algebras on Riemann surfaces. Funktional Anal. i Prilozhen. 27, No.4 (1993), 54-62.

[62] Sheinman, O.K., Modules with highest weight for affine Lie algebras on Riemann surfaces, Funktional Anal. i Prilozhen. 29, No.1 (1995), 44-55

[63] Sheinman, O.K., The fermion model of representations of affine Krichever-Novikov algebras. Funktional Anal. i Prilozhen. 35, No.3 (2001), 209-219.

[64] Sheinman, O.K., Second order casimirs for the affine Krichever-Novikov algebras $\widehat{\mathfrak{g l}}_{g, 2}$ and $\widehat{\mathfrak{s l}}_{g, 2}$, Moscow Math. J. 1 (2001), 605-628.

[65] Sheinman, O.K., Lax operator algebras and Hamiltonian integrable hierarchies, Russ. Math. Surv. 66 (2011), 145-171.

[66] Sheinman, O.K., Current Algebras on Riemann surfaces, Expositions in Mathematics, Vol. 58, De Gruyter, 2012.

[67] A. Tsuchiya, K. Ueno, Y. Yamada, Conformal field theory on universal family of stable curves with gauge symmetries. Adv. Stud. Pure Math. 19 (1989), 459-566.

[68] Tyurin, A.N., Classification of vector bundles on an algebraic curve of an arbitrary genus. Soviet Izvestia, ser. Math., 29, 657-688.

[69] Wagemann, F., Some remarks on the cohomology of Krichever-Novikov algebras, Lett. Math. Phys. 47 (1999), 173-177, Erratum: Lett. Math. Phys. 52 (2000), 349

[70] Wagemann, Friedrich More remarks on the cohomology of Krichever-Novikov algebras. Afr. Mat. 23, No. 1, 41-52 (2012).

University of LuXembourg, Mathematics Research Unit, FSTC, CAMPUS KirChBERG, 6, RUE COUDENHOVE-KALERGI, L-1359 LUXEMBOURG-KIRCHBERG, LUXEMBOURG

E-mail address: martin.schlichenmaiereuni.lu 